\title{
ESTIMATES OF GLOMERULAR FILTRATION RATE BY THE CHRONIC KIDNEY DISEASE EPIDEMIOLOGY COLLABORATION EQUATIONS BEST CORRELATES WITH KINETIC ESTIMATES OF GLOMERULAR FILTRATION RATES IN THE CRITICALLY ILL
}

\author{
Azrina Md Ralib ${ }^{1}$, Farah Nadia Mohd Hanafiah ${ }^{1}$, Fatimah Dzaharudin², Muhammad \\ Rasydan Abd Ghani ${ }^{1}$, Mohd Nizamudin Ismail ${ }^{1}$ and Mohd Basri Mat Nor ${ }^{1}$ \\ ${ }^{1}$ Department of Anaesthesiology and Intensive Care, Kulliyyah of Medicine, \\ International Islamic University Malaysia, Pahang, Malaysia, ${ }^{2}$ Department of \\ Mechanical Engineering, Universiti Malaysia Pahang, Pahang, Malaysia \\ Presenter: Farah Nadia Mohd Hanafiah, mohdhanf@tcd.ie
}

Introduction: Kinetic estimate of GFR (keGFR) is a more accurate estimate of GFR in the acute settings with rapidly changing kidney functions. It takes into account the changes of creatinine over time, creatinine production rate, and the volume of distribution, however needs serial measurement of creatinine. We evaluated which methods of the conventional eGFR measurement best correlates with keGFR. This could assist clinicians in using a simpler method of calculation and is useful in the absence of serial plasma creatinine.

Materials and method: This is a secondary analysis of prospective observational study. Inclusion criteria includes age more than 18 years old, and exclusion criteria includes ICU admission of less than 48 hours, post elective surgery, and ICU readmission. Plasma creatinine were measured daily for five days, and eGFR were calculated using the Cockcroft-Gault, MDRD, CKD-EPI and keGFR equations.

Results: One hundred and forty three patients were analysed, of which 78(54.5\%) had AKI. keGFR strongly correlated with eGFR CKD-EPI $_{\text {at }}$ all time points (all $r \geq 0.90, p<0.0001$ ). The correlation persisted in both AKI and No AKI patients. keGFR only correlated well with eGFR $R_{M D R D}$ and eGFR $R_{C G}$ in AKI patients but less in patients without AKI. Bland Altman analysis showed that eGFR $R_{M D R D}$ had the least bias, but eGFR CKD-EPI $_{\text {had }}$ the greatest precision.

Conclusion: The new equation, keGFR strongly correlated with the eGFR by the CKD-EPI equation regardless of the time points and AKI status, and had the greatest precision. In the absence of serial plasma creatinine measurement, eGFR can accurately be estimated by the CKD-EPI equation compared to others. 